\title{
Comparação de Seis Métodos de Filetagem, em Relação ao Rendimento de Filé e de Subprodutos do Processamento da Tilápia-do-Nilo (Oreochromis niloticus)
}

\author{
Maria Luiza Rodrigues de Souza ${ }^{1}$
}

\begin{abstract}
RESUMO - O objetivo deste experimento foi avaliar seis métodos de filetagem de tilápia-do-nilo (Oreochromis niloticus), considerando-se o rendimento de filé (RFI); dos músculos abdominal (RMA) e hipaxial (RMH); dos resíduos (PRE); da pele bruta (PPB) e limpa (PPL) e do descarne (PDE). Os métodos utilizados foram: $\mathrm{F}_{1}=$ filetagem do peixe inteiro e retirada da pele do filé com a faca (IFP); $\mathrm{F}_{2}=$ retirada da pele do peixe inteiro com alicate e filetagem (IPF); $\mathrm{F}_{3}=$ peixe decapitado e filetagem, com posterior remoção da pele do filé com a faca (CFP); $\mathrm{F}_{4}=$ peixe decapitado, com remoção da pele com auxílio de alicate seguida da filetagem $(\mathrm{CPF}) ; \mathrm{F}_{5}=$ peixe decapitado, removidas as nadadeiras, filetagem e retirada da pele com a faca (CNFP); $\mathrm{F}_{6}=$ peixe decapitado, removidas as nadadeiras, com remoção da pele com alicate e filetagem (CNPF). Foram utilizadas 20 tilápias por tratamento, num delineamento inteiramente casualizado. O método de filetagem influenciou em todos os rendimentos analisados. O método que proporcionou maior rendimento de filé e de músculo hipaxial foi o IPF (36,58\% e 4,22\%, respectivamente) e o pior o CFP (33,66\% e 3,31\%, respectivamente). Para o músculo abdominal, os melhores métodos foram: os CNFP (2,00\%) e CNPF (2,02\%). Quanto aos resíduos, o método que apresentou o melhor resultado foi o IPF (57,96\%), enquanto para pele limpa e descarne o melhor foi o CPF (4,14\% e 45,52\%, respectivamente), apesar de o método IPF (4,00\% e 46,11\%) apresentar resultados muito próximos deste.
\end{abstract}

Palavras-chave: filetagem, Oreochromis niloticus, rendimento de filetagem, tecnologia de pescado

\section{Comparison of Six Filleting Methods and their Relation with Fillet Yield and By-products of Nile Tilapia (Oreochromis niloticus) Processing}

\begin{abstract}
Current experiment evaluates six filleting methods for Nile tilapia with regard to fillet yield (FIY), ventral abdominal muscles (AMY), hypaxial muscles (HMY), residues (REY), crude skin (CSY), clean skin (CSY) and fleshed (FEY). Methods consisted of: $\mathrm{F}_{1}=$ filleting of whole fish and skinning of the fillet with knife, (WFS); $\mathrm{F}_{2}=$ skinning of whole fish with pliers and filleting (WSF); $\mathrm{F}_{3}=$ headless fish and filleting; skinning of fillet with knife (HFS); $\mathrm{F}_{4}=$ headless fish, skinning with pliers and filleting (HSF); $\mathrm{F}_{5}=$ headless fish; removal of fins; filleting and skinning with knife (HFFS); $\mathrm{F}_{6}=$ headless fish; removal of fins, skinning with pliers and filleting (HFSF). Twenty tilapias were used for each treatment in a completely randomized design. Filleting method affected all analyzed yield. WSF had the best fillet yield and hypaxial muscle (36.58\% and $4.22 \%$, respectively); lowest yield was provided by HFS (33.66\% and $3.31 \%$, respectively). With regard to abdominal muscle, HFFS (2.00\%) and HFSF (2.02\%) were the best. In the case of residues, WSF gave the best result (57.96\%); HSF was the best method for clean skin and fleshing ( $4.14 \%$ and $45.52 \%$, respectively), with WSF $(4,00 \%$ and $46.11 \%)$ as next to best result.
\end{abstract}

Key Words: fillet processing, Oreochromis niloticus, fillet yield, fish technology

\section{Introdução}

A aqüicultura é uma alternativa para incrementar os índices de consumo de proteínas de origem animal e um importante fator de desenvolvimento socio-econômico para o país. Atualmente, apesar das crises econômicas e do surgimento de novas enfermidades, a aqüicultura é considerada um dos sistemas de produção de alimentos que mais cresce no mundo, e que poderá contribuir muito com a crescente demanda mundial de pescado neste milênio.
Não obstante, a aqüicultura ainda apresenta deficiências em relação à falta de padronização do produto para o consumidor, o que acarreta dificuldades quanto às características de sabor, presença ou não de espinhas, forma de preparo e valor nutricional. Entretanto, se o produto tiver boa apresentação (postas ou filé) e embalagem (com especificação do produto), torna-se mais fácil o trabalho de marketing e, conseqüentemente, a colocação do pescado no mercado. Sem dúvida, a procura por um alimento de qualidade e de fácil preparo é uma das maiores estratégias de marketing exploradas por indústrias de alimentos. 
A Oreochromis niloticus, segundo Vannuccini (1999), tem sido etiquetada como o "novo pescado branco". Esta espécie apresenta os requisitos típicos dos peixes preferidos pelo mercado consumidor, tais como carne branca de textura firme, sabor delicado e fácil filetagem, não tendo espinha em "Y" nem odor desagradável. Possui além disso, as características que a colocam no pódio das principais espécies cultivadas comercialmente, as quais, de acordo com Kubitza \& Kubitza (2000), são: a facilidade de reprodução e obtenção de alevinos, a possibilidade de manipulação hormonal do sexo para obtenção dos machos, aceitação de diversos alimentos e capacidade de aproveitar alimentos naturais em viveiros; conversão alimentar entre 1 e 1,6; excelente crescimento em cultivo intensivo; grande rusticidade (manejo intenso e baixos níveis de oxigênio dissolvido) e resistência a doenças.

A tilápia é atualmente a segunda espécie mais cultivada mundialmente (Jory et al., 2000). Os mesmos autores ainda relatam que ela se converteu rapidamente em um dos produtos mais populares no mercado de produtos aquáticos dos Estados Unidos, tanto em volume como na diversidade de apresentação (tilápia eviscerada fresca e congelada, inteira e em filés). A prova disso é o aumento na importação ocorrida nos últimos anos, o qual, em peixe inteiro congelado, foi de 12.062 para 21.535 toneladas, e em filés frescos, de 1.460 para 3.590 toneladas, isso de 1995 para 1998. A importação dos filés congelados, em 1998, chegou a 2.696 toneladas (Jory et al., 2000). O filé também é comercializado com pele; no entanto, a porcentagem de comercialização nessa forma de processamento é reduzida (Delfini, 2001) ${ }^{*}$.

Segundo Castillo Campo (2001), os Estados Unidos, em 2000, importaram 40.469 toneladas de tilápia, sendo 27.781 toneladas de peixe inteiro congelado, 5.185 de filé congelado e 7.501 de filé fresco. O mesmo autor menciona que o consumo de tilápia, somadas e produzida no país e a importada, foi de 90.720 toneladas em peso vivo, no final do ano 2000 , enquanto em 1998 alcançara 50.803 toneladas.

Os principais exportadores de tilápia inteira e filés congelados são os países asiáticos, como a Tailândia, Taiwan e Indonésia; e de filés frescos, países latino-americanos como a Costa Rica, o Equador e Honduras (Jory et al., 2000). Entretanto, existe a expectativa de que o Equador se converta no líder fornecedor de filés frescos de tilápia ao mercado norte-americano, ultrapassando a Costa Rica, onde a produção de tilápia teve um incremento superior a $10 \%$ ao ano (Redmayne, 2000). Em relação ao Brasil, em 1995, a produção de pescado advindo da aqüicultura foi ao redor de 27.250 toneladas, o que colocava o Brasil em trigésimo terceiro entre os principais países que praticam a aqüicultura (Hilsdorf \& Pereira, 1999). De acordo com Kubitza (2000), a produção anual de tilápia está entre 30 e 40 mil toneladas.

Considerando-se esse crescimento na produção de tilápia, é interessante conhecer as formas de comercialização e os métodos aplicados para obtenção do filé, já que o aumento nesta forma de apresentação do produto final vem crescendo muito nos últimos anos. Além disso, é importante identificar o método de filetagem que apresenta os melhores resultados. Por não existir um padrão de filetagem, há divergência em relação ao melhor método a ser empregado, ou seja, qual método proporciona o maior rendimento de filé, facilidade operacional e menor tempo de processamento.

O objetivo do experimento foi analisar seis métodos de filetagem utilizados em unidades de beneficiamento de tilápia-do-nilo (Oreochromis niloticus) e em pesqueiros, para determinar qual deles proporciona melhores rendimentos das partes comestíveis e a porcentagens de pele e demais resíduos de filetagem.

\section{Material e Métodos}

O experimento foi realizado na Estação de Piscicultura da Universidade Estadual de Maringá/ CODAPAR (PR-Brasil). Foram utilizados 120 exemplares de tilápia-do-nilo (Oreochromis niloticus), com peso médio de 362,66 g e comprimento-padrão médio de 20,85 cm, capturados em tanques de depuração, por meio de puçás, e abatidos por destruição da medula espinhal. Após o abate, foram pesados em balança de precisão de $0,1 \mathrm{~g}$ e, em seguida, filetados de acordo com cada tratamento proposto.

A metodologia utilizada baseou-se na retirada ou não da cabeça e nadadeiras do peixe antes do processo de filetagem, e na forma de remover a pele, antes ou depois da remoção do filé. Para tanto, a filetagem foi realizada por uma única pessoa, aplicando-se seis

* DELFINI, A. (Vice-presidente de produção da AQUAMAR S.A., Guayaquil-Equador). Comunicação pessoal, 2001.

R. Bras. Zootec., v.31, n.3, p.1076-1084, 2002 
métodos: $\mathrm{F}_{1}=$ com o peixe inteiro sem eviscerar: faz-se primeiramente a retirada do filé com pele e, em seguida, separa-se esta do filé, com o auxílio de uma faca (IFP), (Figura 1); $F_{2}=$ com peixe inteiro sem eviscerar: retira-se a pele, com auxílio de alicate especial, e depois o filé (IPF), (Figura 2); $\mathrm{F}_{3}=$ com o peixe decapitado e eviscerado: faz-se primeiramente a retirada do filé com pele, e depois a pele do filé, com o auxílio de uma faca (CFP), (Figura 3 ); $\mathrm{F}_{4}=$ com o peixe decapitado e eviscerado: retira-se a pele com o auxílio de alicate especial e depois o filé (CPF), (Figura 4); $\mathrm{F}_{5}=$ com o peixe decapitado e eviscerado:

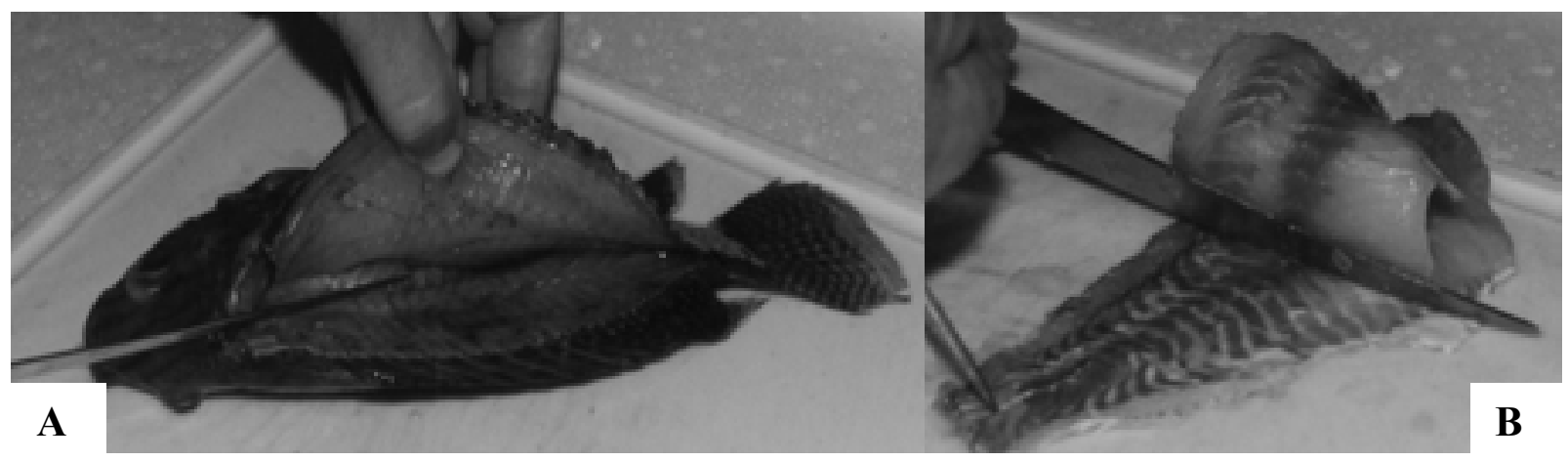

Figura 1 - Método de filetagem $F_{1}$ (IFP): filetagem do peixe inteiro (A) e retirada da pele do filé com faca (B). Figure 1 - Filleting processing $F_{1}$ (WFS): filleting of whole fish $(A)$ and skinning of the fillet with knife $(B)$.

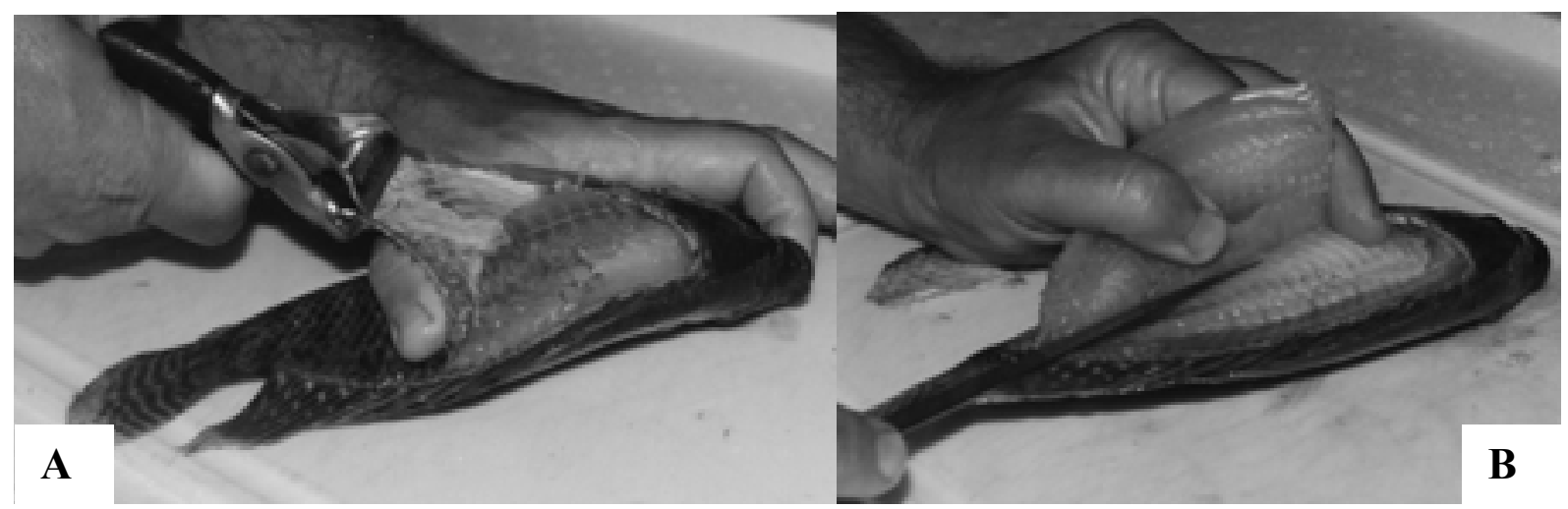

Figura 2 - Método de filetagem $F_{2}$ (IPF): retirada da pele do peixe inteiro com alicate (A) e filetagem (B). Figure 2 - Filleting processing $F_{2}(W S F)$ : skinning of whole fish with pliers $(A)$ and felleting $(B)$.

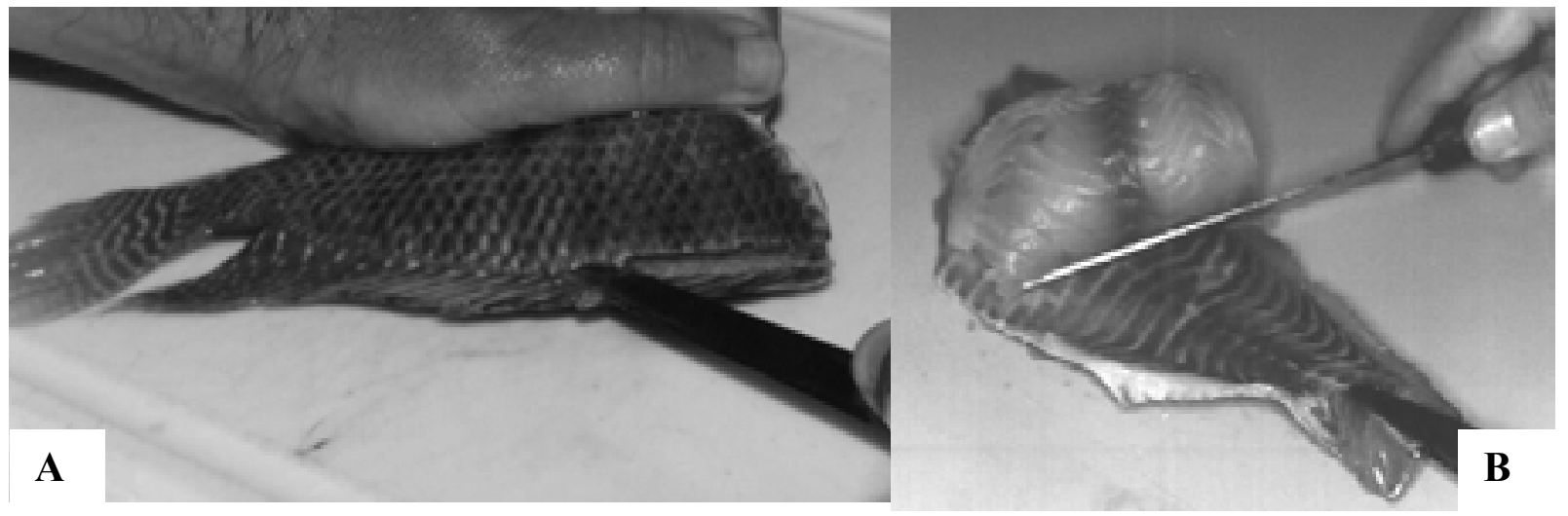

Figura 3 - Método de filetagem $F_{3}(C F P)$ = peixe decapitado, realização da filetagem $(A)$ e, depois, a remoção da pele do filé com a faca (B).

Figure 3 - Filleting processing $F_{3}(H F S)$ : headless fish, and the filleting (A); and later the skinning of fillet with knife (B). 
removem-se as nadadeiras (dorsal e anal), faz-se a filetagem (filé com pele), e a seguir, com auxílio de uma faca, remove-se a pele (CNFP), (Figura 5) e $\mathrm{F}_{6}=$ com o peixe decapitado e eviscerado: removem-se as nada-

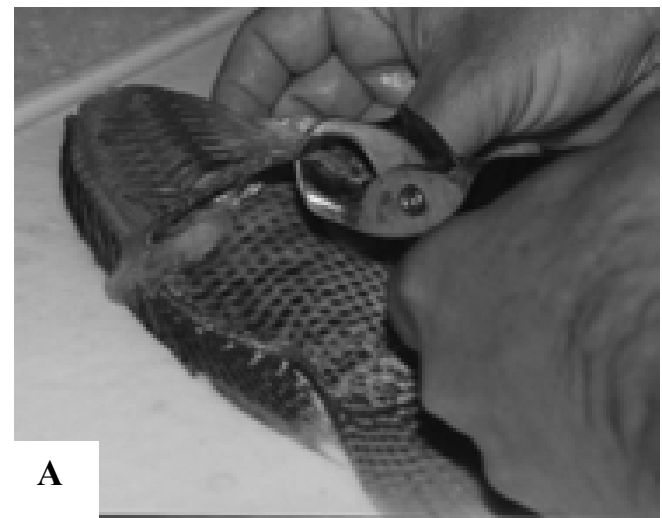

deiras (dorsal e anal), faz-se a retirada da pele com alicate especial, e realiza-se a filetagem (filé sem pele), (CNPF), (Figura 6). Em todas as situações o filé foi obtido a partir da musculatura dorsal, nas duas

Figura 4 - Método de filetagem $\mathrm{F}_{4}(\mathrm{CPF})$ = peixe decapitado, remoção da pele com auxílio de alicate $(\mathrm{A})$ e, depois, a filetagem (B).

Figure 4 - Filleting processing $F_{4}$ (HSF): headless fish, skinning with pliers $(A)$ and, after, filleting $(B)$.
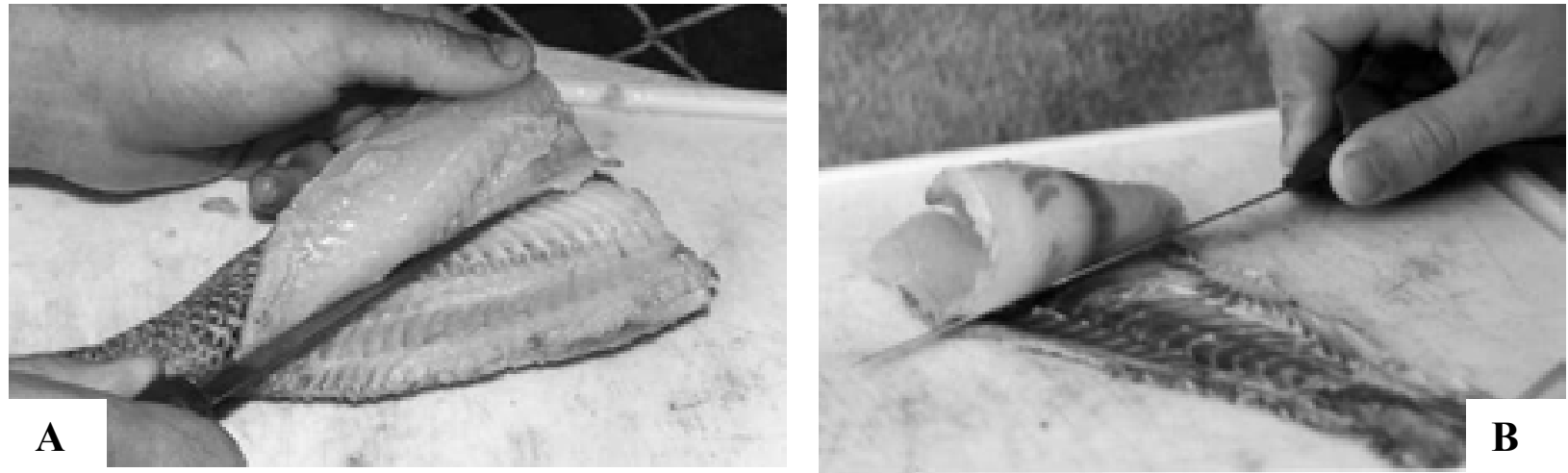

Figura 5 - Método de filetagem $\mathrm{F}_{5}(\mathrm{CNFP})$ = peixe decapitado, remoção das nadadeiras, filetagem $(\mathrm{A})$ e retirada da pele com faca $(\mathrm{B})$.

Figure 5 - Filleting processing $F_{5}$ (HFFS): headless fish, removal of fins, filleting (A) and skinning with knife (B).
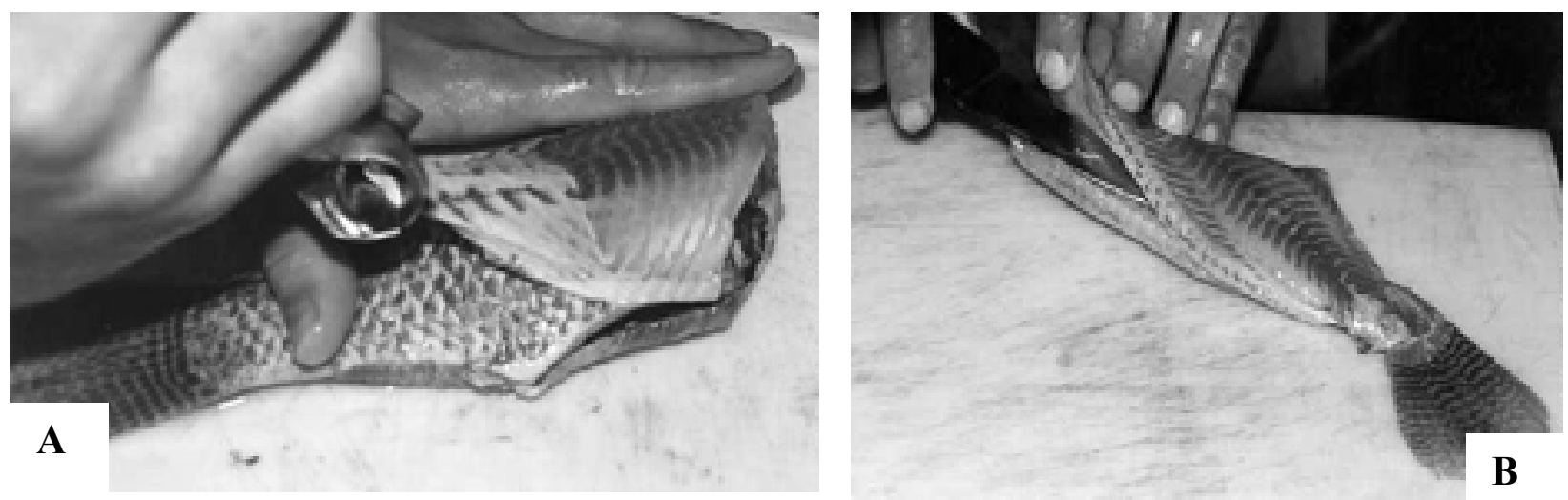

Figura 6 - Método de filetagem $\mathrm{F}_{6}(\mathrm{CNPF})$ = peixe decapitado, remoção das nadadeiras, retirada da pele com alicate $(A)$ e filetagem $(B)$.

Figure 6 - Filleting processing $F_{6}(H F S F)$ : headless fish, removal of fins, skinning with pliers (A) and filleting (B).

R. Bras. Zootec., v.31, n.3, p.1076-1084, 2002 
laterais do peixe no sentido longitudinal, ao longo de toda a extensão da coluna vertebral e costelas.

Após a obtenção do filé, estando o peixe já decapitado, foi removido o músculo hipaxial profundo, localizado entre a câmara gasosa e a cavidade peritoneal (Figura 7A). Para sua obtenção, deve-se realizar um corte, a partir da câmara gasosa em direção à região ventral do peixe, de forma que a faca percorra paralelamente a extensão até a extremidade das costelas (Figura 7A,B). O músculo abdominal

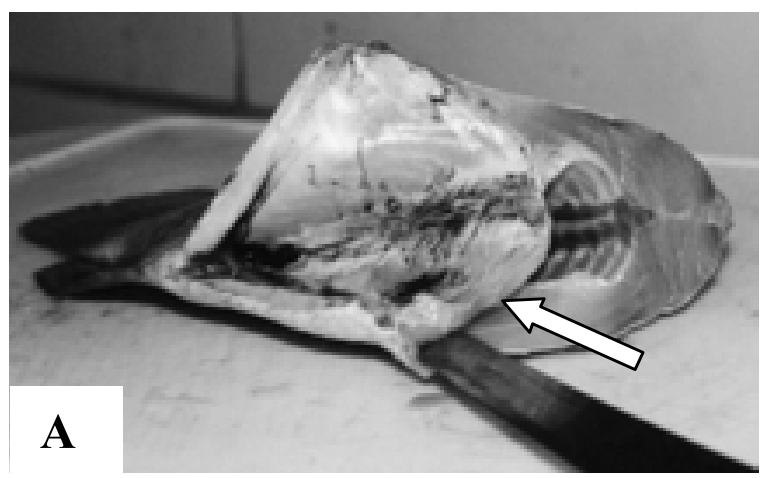

ventral ou "barriguinha" encontra-se logo após o término dessas costelas.

Para análise foram considerados o peso total, comprimento padrão, peso do filé sem pele, músculo abdominal ventral e hipaxial profundo, resíduos (peso total menos as partes comestíveis totais), pele bruta (após a esfola, com escamas e restos de músculos), pele limpa (após escamar e descarnar) e descarne (resíduos = escama, músculos e tecido adiposo referente ao peso bruto da pele). Todos os dados de

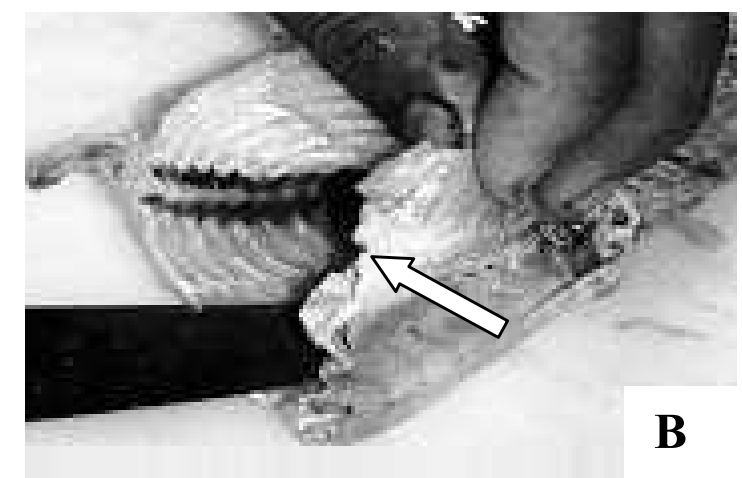

Figura 7 - Localização do músculo hipaxial profundo $(A)$ e corte do músculo após filetagem (B), (seta). Figure 7 - Localization of deep hypaxial muscle $(A)$ and cut in the muscle after filleting (B), (arrow).

rendimento foram calculados em função do peso total do exemplar.

O delineamento experimental foi o inteiramente casualizado, com seis tratamentos, 20 repetições, sendo cada peixe considerado uma parcela experimental.

Os dados obtidos foram submetidos à análise de variância e as médias, comparadas pelo teste de Tukey, em nível de 5\% de probabilidade (Banzatto \& Kronka, 1995). O modelo matemático utilizado foi:

$$
\begin{gathered}
Y_{i j}=\mu+F_{i}+e_{i j} \\
(i=1,2, \ldots, 6 ; j=1,2, \ldots, 20)
\end{gathered}
$$

em que: $Y_{i j}=$ valor observado no peixe $j$, com o método de filetagem $\mathrm{i} ; \mu=$ média; $\mathrm{F}_{\mathrm{i}}=$ efeito do método de filetagem $\mathrm{i}$; e $\mathrm{e}_{\mathrm{ij}}=$ erro experimental.

\section{Resultados e Discussão}

Observa-se na Tabela 1 que não houve diferença significativa para os valores médios do peso corporal (g) e o comprimento-padrão (cm) dos peixes utilizados para análise dos métodos de filetagem, confirmando que os peixes utilizados eram homogêneos, em relação ao tamanho.

\section{R. Bras. Zootec., v.31, n.3, p.1076-1084, 2002}

Comparando-se os métodos de filetagem quanto às características de peso, houve diferença significativa $(\mathrm{P}<0,01)$ entre os tratamentos para os músculos abdominal ventral e hipaxial profundo e para a pele limpa; entretanto, no peso do filé, dos resíduos totais, da pele bruta e do descarne os métodos analisados não influenciaram $(\mathrm{P}>0,05)$ (Tabelas $1 \mathrm{e} 2)$. Por outro lado, os métodos de filetagem influenciaram nas características de rendimento de todas as variáveis analisadas, conforme Tabelas 1 e 2 .

Em um processo de filetagem, o rendimento de filé é o aspecto mais importante, embora seja interessante conhecer o seu peso. Por exemplo, atualmente nos Estados Unidos, o consumidor tem preferência pelo filé de tilápia em duas classificações de peso, que são "cinco a sete onzas" (correspondentes a um filé de $141 \mathrm{~g}$ a $198 \mathrm{~g}$ ) e "sete a nove onzas" (198 a $255 \mathrm{~g}$ ), (Castillo Campo, 2001).

Observa-se, na Tabela 1, que não ocorreu diferença significativa no peso; porém comparando-se os tratamentos IFP e IPF; CFP e CPF e, o CNFP e CNPF, pode-se verificar que os maiores valores foram para os métodos em que foi retirada a pele e 
depois realizada a filetagem $(\mathrm{IPF}=131,31 \mathrm{~g} ; \mathrm{CPF}=$ $123,72 \mathrm{~g}$ e $\mathrm{CNPF}=128,80 \mathrm{~g}$ ).

O método de filetagem influenciou sobre o rendimento do filé, onde o método IPF $(36,58 \%)$ proporcionou uma maior porcentagem de filé e o CFP $(33,66$ \%) a menor, enquanto os demais tratamentos não diferiram entre si. De acordo com Contreras-Guzmán (1994), o rendimento de filé com pele, de espécies marinhas e de água doce, encontra-se entre $32,8 \%$ e $59,8 \%$, com uma média de 50,5\%; no entanto, com a remoção da pele, o rendimento de filé reduz para $43 \%$. Não obstante, na literatura observam-se resultados de rendimento de filé para tilápia-do-nilo que variam de 25,4\% a 42\% (Clement \& Lovell, 1994; ContrerasGuzmán, 2002), em relação ao peso bruto do peixe.

Ribeiro et al. (1998) obtiveram um rendimento em torno de $31,89 \%$, para tilápia-do-nilo com peso entre 351 e $550 \mathrm{~g}$, valor bem inferior aos obtidos neste experimento. Esses mesmos autores realizaram a filetagem e depois removeram a pele da musculatura utilizando-se de faca. Pela abordagem realizada por Ribeiro et al. (1998), os peixes não foram decapitados e eviscerados, portanto corresponderam ao método
IFP, deste experimento, cujo rendimento de filé foi de $34,58 \%$, conseqüentemente, superior ao obtido pelos autores citados anteriormente. Souza et al. (1998) abatendo a mesma espécie com peso médio entre 395 e $530 \mathrm{~g}$, obtiveram os valores entre 32,09 e $37,14 \%$, portanto, maior variação na porcentagem de filé. Souza \& Maranhão (2001) relatam para tilápias-donilo de 300 a $500 \mathrm{~g}$ de peso corporal um rendimento de filé de 36,50 a $36,84 \%$, quando submetidas ao método de filetagem realizado em série por mais de uma pessoa, em que os peixes foram primeiramente eviscerados, foram retiradas as peles e somente depois foram filetados, correspondendo ao tratamento IPF, do presente estudo. Os valores obtidos neste experimento estão de acordo com os dos autores mencionados.

Neste experimento dos métodos de filetagem, a decapitação foi realizada de forma oblíqua ou inclinada, em peixes que apresentavam pesos dentro da mesma faixa estudada por Souza et al. (2000), para a tilápiado-nilo entre 250 e $400 \mathrm{~g}$. Esses autores analisaram a influência do tipo de corte da cabeça (decapitação) sobre o rendimento de filé. Obtiveram um rendimento de filé de 32,$64 ; 31,86$ e 27,72\% em função dos tipos

Tabela 1 - Valores médios do peso corporal $(\mathrm{g})$, comprimento padrão $(\mathrm{cm})$, pesos $(\mathrm{g})$ e rendimentos $(\%)$ dos cortes do processamento de filetagem, comparando seis métodos de filetagem

Table 1 - Mean values body weight $(\mathrm{g})$, standard lenght $(\mathrm{cm})$, weight $(\mathrm{g})$ and yied $(\%)$ to cut of filleting processing, comparation six filleting methods

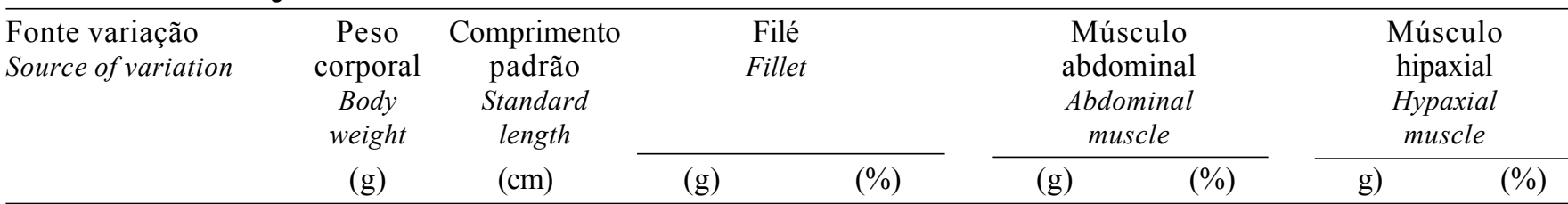

Métodos de filetagem

Filleting processing

\begin{tabular}{|c|c|c|c|c|c|c|c|c|}
\hline $\mathrm{F}_{1}$-IFP & $366,40^{\mathrm{a}}$ & $21,13^{\mathrm{a}}$ & $126,10^{\mathrm{a}}$ & $34,58^{\mathrm{ab}}$ & $6,05^{\mathrm{abc}}$ & $1,68^{\mathrm{ab}}$ & $11,13^{b c}$ & $3,05^{b c}$ \\
\hline $\mathrm{F}_{2}$-IPF & $359,80^{\mathrm{a}}$ & $20,74^{\mathrm{a}}$ & $131,31^{\mathrm{a}}$ & $36,58^{a}$ & $4,42^{\mathrm{d}}$ & $1,23^{\mathrm{c}}$ & $15,26^{\mathrm{a}}$ & $4,22^{\mathrm{a}}$ \\
\hline $\begin{array}{l}F_{2}-\mathrm{WSF} \\
\mathrm{F}_{3}-\mathrm{CFP} \\
F_{2}-H F S\end{array}$ & $354,30^{\mathrm{a}}$ & $20,88^{a}$ & $118,89^{a}$ & $33,66^{\mathrm{b}}$ & $5,88^{b c d}$ & $1,67^{\mathrm{abc}}$ & $11,84^{\mathrm{b}}$ & $3,31^{\mathrm{b}}$ \\
\hline $\mathrm{F}_{4}-\mathrm{CPF}$ & $357,80^{\mathrm{a}}$ & $20,76^{\mathrm{a}}$ & $123,72^{\mathrm{a}}$ & $34,54^{\mathrm{ab}}$ & $5,52^{\mathrm{cd}}$ & $1,55^{\mathrm{bc}}$ & $8,28^{\mathrm{c}}$ & $2,32^{\mathrm{c}}$ \\
\hline $\begin{array}{l}\mathrm{F}_{5} \text {-CNFP } \\
F_{5}-H F F S\end{array}$ & $365,95^{a}$ & $20,69^{a}$ & $127,95^{\mathrm{a}}$ & $34,94^{\mathrm{ab}}$ & $7,30^{\mathrm{ab}}$ & $2,00^{\mathrm{a}}$ & $11,40^{\mathrm{b}}$ & $3,12^{\mathrm{b}}$ \\
\hline $\begin{array}{l}\mathrm{F}_{6}-\mathrm{CNPF} \\
F_{6}-H F S F\end{array}$ & $371,70^{\mathrm{a}}$ & $20,94^{\mathrm{a}}$ & $128,80^{\mathrm{a}}$ & $34,63^{\mathrm{ab}}$ & $7,50^{\mathrm{a}}$ & $2,02^{\mathrm{a}}$ & $9,60^{\mathrm{bc}}$ & $2,59^{b c}$ \\
\hline $\begin{array}{l}\text { Teste } \mathrm{F} \\
\text { F Test }\end{array}$ & $045^{\mathrm{ns}}$ & $0,48^{\mathrm{ns}}$ & $1,38^{\mathrm{ns}}$ & $2,68^{*}$ & $8,84 * *$ & $7,30 * *$ & $9,98 * *$ & $12,37 * *$ \\
\hline CV $(\%)$ & 11,91 & 5,10 & 13,19 & 7,54 & 28,31 & 28,85 & 29,80 & 27,02 \\
\hline
\end{tabular}

a,b,c,d - em cada coluna, médias seguidas de mesma letra não diferem entre si, pelo teste de Tukey $(P>0,05) ;$ ns - não significativo $(P>0,05) *$ - significativo $(P<0,05) * *$ - significativo $(P<0,01)$

$a, b$ - in each column, means followed by the same letters, did not differ by Tukey test $(P>0.05)$ ns - not significant $(P>0.05)$ * - significant $(P<0.05) \quad * *$ significant $(P<0.01)$

\section{R. Bras. Zootec., v.31, n.3, p.1076-1084, 2002}


de corte oblíquo, contornado e reto, respectivamente. Os resultados observados neste experimento, para rendimento de filé, foram superiores, se comparados aos do mesmo método de corte de cabeça relatado por Souza et al. (2000).

Souza et al. (1999) obtiveram rendimentos de 32,89 e $36,67 \%$ para filé de tilápias-do-nilo submetidas aos métodos de filetagem remoção do filé e depois retirada da pele do filé e retirada da pele com alicate e filé, os quais corresponderam aos métodos IFP e IPF deste experimento, cujos valores foram, respectivamente, 34,58 e $36,58 \%$. O resultado obtido no método IPF deste experimento foi semelhante ao observado por Souza et al. (1999).

Em relação ao músculo abdominal, o método que proporcionou maior peso foi o $\operatorname{CNPF}(7,50 \mathrm{~g})$, não diferindo do CNFP (7,30 g) e IFP (6,05 g); enquanto o que apresentou o menor peso foi o IPF $(4,42 \mathrm{~g})$, que por sua vez não diferiu de $\operatorname{CFP}(5,88 \mathrm{~g})$ e $\operatorname{CPF}(5,52 \mathrm{~g})$. Todavia, quanto ao músculo hipaxial profundo foi o método IPF $(15,26 \mathrm{~g})$ o que apresentou o maior peso; e o menor foi o de CPF $(8,28 \mathrm{~g})$. O fato de o músculo abdominal ventral ter sido menor no IPF explica o porquê de se obter um maior valor para o músculo hipaxial profundo, pois para a remoção deste músculo do peixe é realizado um corte ventralmente próximo às costelas em direção à região ventral, e caso o corte do músculo ventral tenha sido menor, restará maior quantidade para o músculo hipaxial.

Para o músculo abdominal, o menor rendimento foi verificado no método IPF $(1,23 \%)$, apesar de não ter diferido significativamente de CFP $(1,67 \%)$ e CPF (1,55\%); enquanto os melhores rendimentos de músculo abdominal foram observados para CNPF $(2,02 \%)$ e CNFP (2,00\%), não diferindo de IFP $(1,68 \%)$ e CFP (1,67\%). O músculo hipaxial profundo apresentou maior porcentagem no método IPF $(4,22 \%)$ e menor no CPF (2,32\%). Os valores de rendimento do músculo abdominal ventral obtidos neste experimento, analisando-se os diferentes métodos de filetagem, foram inferiores (variando entre 1,23 e $2,02 \%$ ) aos obtidos por Souza \& Maranhão (2001), para tilápias-do-nilo com peso de 300 a 400 g e de 401 a $500 \mathrm{~g}$ de peso corporal, cujos rendimentos foram 3,17 e $3,51 \%$, respectivamente. Souza et al. (2001) citaram rendimentos de 2,45 e 2,85\% para o músculo abdominal ventral e 3,75 e $4,72 \%$ para o músculo hipaxial profundo, respectivamente, para tilápias-donilo com pesos corporais de 250 a $400 \mathrm{~g}$ e de 401 a $550 \mathrm{~g}$. Porcentagem superior de músculo abdominal (4,56\%) foi obtida por Marengoni et al. (1998) para tilápia com peso de 613,93 g; e Pereira \& Campos (2000) relatam porcentagem de barriga (músculo ventral) de $3 \%$, para tilápias de $500 \mathrm{~g}$.

Tabela 2 - Valores dos pesos (g) e rendimentos (\%) dos resíduos do processamento da tilápia-do-nilo, em seis métodos de filetagem

Table 2 - Mean values weight $(g)$ and yied (\%) of residues of processing of Nile tilapia, in six filleting methods

\begin{tabular}{|c|c|c|c|c|c|c|c|c|}
\hline \multirow[t]{2}{*}{$\begin{array}{l}\text { Fonte variação } \\
\text { Source of variation }\end{array}$} & \multicolumn{2}{|c|}{$\begin{array}{l}\text { Resíduo totais } \\
\text { Total residue }\end{array}$} & \multicolumn{2}{|c|}{$\begin{array}{l}\text { Pele Bruta } \\
\text { Crude skin }\end{array}$} & \multicolumn{2}{|c|}{$\begin{array}{l}\text { Pele limpa } \\
\text { Clean skin }\end{array}$} & \multicolumn{2}{|c|}{$\begin{array}{c}\text { Descarne } \\
\text { Fleshed }\end{array}$} \\
\hline & (g) & $(\%)$ & (g) & $(\%)$ & (g) & $(\%)$ & (g) & $(\%)$ \\
\hline $\begin{array}{l}\mathrm{F}_{1} \text {-IFP } \\
F_{1} \text {-WFS }\end{array}$ & $223,13^{a}$ & $60,69^{a}$ & $26,90^{\mathrm{a}}$ & $7,43^{\mathrm{a}}$ & $12,65^{\mathrm{abc}}$ & $3,46^{\mathrm{bc}}$ & $14,35^{\mathrm{a}}$ & $53,17^{\mathrm{a}}$ \\
\hline $\begin{array}{l}\mathrm{F}_{2}-\mathrm{IPF} \\
F_{2}-W S F\end{array}$ & $208,81^{\mathrm{a}}$ & $57,96^{\mathrm{b}}$ & $26,80^{\mathrm{a}}$ & $7,48^{\mathrm{a}}$ & $14,40^{\mathrm{ab}}$ & $4,00^{\mathrm{ab}}$ & $12,40^{\mathrm{a}}$ & $46,11^{\mathrm{ab}}$ \\
\hline $\begin{array}{l}\mathrm{F}_{3}-\mathrm{CFP} \\
F_{3}-H F S\end{array}$ & $217,70^{\mathrm{a}}$ & $61,36^{\mathrm{a}}$ & $27,50^{\mathrm{a}}$ & $7,73^{\mathrm{a}}$ & $13,55^{\mathrm{abc}}$ & $3,82^{\mathrm{abc}}$ & $14,10^{\mathrm{a}}$ & $49,95^{\mathrm{ab}}$ \\
\hline $\begin{array}{l}\mathrm{F}_{4}-\mathrm{CPF} \\
F_{4}-H S F\end{array}$ & $220,29^{a}$ & $61,59^{\mathrm{a}}$ & $27,50^{\mathrm{a}}$ & $7,67^{\mathrm{a}}$ & $14,80^{\mathrm{a}}$ & $4,14^{\mathrm{a}}$ & $12,70^{\mathrm{a}}$ & $45,52^{b}$ \\
\hline $\begin{array}{l}\mathrm{F}_{5}-\mathrm{CNFP} \\
F_{5}-H F F S\end{array}$ & $219,30^{\mathrm{a}}$ & $59,93^{\mathrm{ab}}$ & $25,70^{\mathrm{a}}$ & $6,98^{\mathrm{ab}}$ & $11,85^{\mathrm{c}}$ & $3,26^{\mathrm{c}}$ & $13,85^{\mathrm{a}}$ & $52,69^{\mathrm{ab}}$ \\
\hline $\begin{array}{l}\mathrm{F}_{6}-\mathrm{CNPF} \\
F_{6}-H F S F\end{array}$ & $225,80^{\mathrm{a}}$ & $60,76^{\mathrm{a}}$ & $23,40^{\mathrm{a}}$ & $6,31^{\mathrm{b}}$ & $12,50^{\mathrm{bc}}$ & $3,34^{\mathrm{c}}$ & $11,00^{\mathrm{a}}$ & $46,41^{\mathrm{ab}}$ \\
\hline $\begin{array}{l}\text { Teste } \mathrm{F} \\
\text { F test }\end{array}$ & $0,77^{\mathrm{ns}}$ & $3,97 * *$ & $2,02^{\mathrm{ns}}$ & $4,23 * *$ & $4,54 * *$ & $6,48 * *$ & $1,93^{\mathrm{ns}}$ & $3,62 * *$ \\
\hline C.V.(\%) & 13,61 & 4,90 & 18,72 & 16,12 & 18,25 & 17,53 & 31,50 & 16,49 \\
\hline
\end{tabular}

a,b - em cada coluna, médias seguidas de mesma letra não diferem entre si, pelo teste de Tukey $(P>0,05)$ ns - não significativo $(P>0,05)$ $* *$ - significativo $(P<0,01)$.

$a, b$ - in each column, means followed of same letters, did not differ among thenselves by Tukey test $(P>0.05)$ ns - non significant $(P>0.05) * *-s i g n i f i c a n t$ $(P<0.01)$.

\section{R. Bras. Zootec., v.31, n.3, p.1076-1084, 2002}


Como os peixes deste experimento estão numa mesma categoria de peso, as diferenças significativas observadas atribuem-se ao tipo de corte realizado para obtenção do músculo abdominal e hipaxial. Portanto, tais diferenças devem-se à falta de padronização dos cortes e experiência do filetador. Porém, analisando-se todos os aspectos que possam influenciar nos rendimentos de músculo abdominal ventral e hipaxial profundo, pode-se dizer que o peso do peixe, o método de filetagem, a destreza do filetador e a determinação da forma de corte dos músculos influenciam no rendimento desses dois músculos analisados.

Em relação aos resíduos totais, o método IPF apresentou a melhor porcentagem (57,96\%), ou seja, a menor proporção a ser descartada (Tabela 2), não diferindo de $(\mathrm{CNFP}=59,93 \%)$, enquanto este tratamento não diferiu dos demais. Portanto, o método de filetagem IPF permite melhor aproveitamento das partes comestíveis da tilápia-do-nilo. Os valores obtidos para resíduos totais foram inferiores aos relatados por Marchi (1997), para tilápia-do-nilo (380 $\mathrm{g}$ de peso corporal), que foi de 67\%, e Makrakis et al. (2000), que variou de 65,94 a $72,31 \%$.

Pode-se observar na Tabela 2 que, apesar de não ter ocorrido diferença no peso da pele bruta entre os métodos de filetagem, ou seja, de não ter havido efeito do corte da cabeça, nadadeiras ou remoção da pele sobre essa variável analisada, os menores pesos e porcentagens observados entre os tratamentos foram observados para os tratamentos CNFP $(25,70 \mathrm{~g}$ e $6,98 \%$, respectivamente) e CNPF $(23,40 \mathrm{~g}$ e $6,31 \%$, respectivamente). Quando as peles brutas foram submetidas à limpeza (pele limpa), observou-se diferença significativa nos tratamentos, com relação à remoção das nadadeiras, com resultados inferiores, tanto no peso quanto no rendimento $(\mathrm{CNFP}=11,85 \mathrm{~g} ; 3,26 \% \mathrm{e} \mathrm{CNPF}=$ $12,50 \mathrm{~g} ; 3,34 \%$, respectivamente) para aqueles dos quais não foram retiradas as nadadeiras, apesar de não ter ocorrido diferença entre estes e o IFP $(12,65 \mathrm{~g}$; $3,46 \%)$ e CFP $(13,55 \mathrm{~g} ; 3,82 \%)$. A pele limpa no método $\mathrm{CPF}$ apresentou o maior peso $(\mathrm{CPF}=14,80 \mathrm{~g})$ e conseqüentemente, um resultado superior para rendimento $(4,14 \%)$, quando comparado aos demais tratamentos, não diferindo de IPF (4,00\%) e CFP (3,82\%). O mesmo método (CPF) apresentou a menor porcentagem de descarne (45,52\%). Portanto, o método CPF proporciona a maior pele para utilização no curtimento, com menor quantidade de partes indesejáveis.

Não ocorreu diferença significativa entre os tratamentos para o descarne, mas as médias foram superiores para os tratamentos com retirada do filé e depois, da pele do filé (IFP=14,35 g; CFP=14,10 g e $\mathrm{CNFP}=13,85 \mathrm{~g}$ ), comparativamente aos métodos em que foram removidos a pele e depois os filés (IPF $=12,40 \mathrm{~g}$; $\mathrm{CPF}=12,70 \mathrm{~g}$ e $\mathrm{CNPF}=11,00 \mathrm{~g}$ ).

Também pode ser observado (Tabela 2) que as porcentagens de descarne ou de remoção de partes indesejadas da pele (maior percentual de escamas e músculos aderidos à pele) foram maiores nos tratamentos onde foram retirados os filés para depois remover o filé da pele, apesar de não ter ocorrido diferença entre o IPF e CNPF.

$\mathrm{Na}$ literatura consultada, não há dados relativos a estas variáveis analisadas, exceto no tocante a peso ou porcentagem de pele bruta ou pele sem escamas, para algumas espécies de peixes. Os valores observados neste trabalho para pele limpa foram de $3,26 \%$ a $4,14 \%$, inferiores aos relatados por Freitas \& Gurgel (1984) para pele sem escama (5\%). Os autores não mencionaram a presença de musculatura na pele, fato ao qual talvez se deva esta maior porcentagem. Em relação à porcentagem de pele bruta, houve variação de $6,31 \%$ a $7,73 \%$ entre os seis métodos de filetagem, corroborando Contreras-Guzmán (1994), o qual cita que a pele perfaz em média $7,5 \%$ do peso dos peixes teleósteos, e Makrakis et al. (2000), que acusam uma variação de 7,28\% a 8,58\%. Todavia, discordam com as porcentagens obtidas por Macedo-Viegas et al. (1997), para a tilápia-do-nilo (250 a 450 g), em relação à pele bruta (variação de $4,77 \%$ a 5,71\%).

Souza et al. (1999) citam porcentagens de descarne variando de $40,63 \%$ a $58,97 \%$, ao compararem dois métodos de filetagem e quatro categorias de peso da tilápia. Os valores obtidos neste experimento dos seis métodos de filetagem estão dentro dos valores obtidos por Souza et al. (1999).

Tornam-se necessárias outras investigações em relação a diferentes métodos de filetagem praticados por unidades de beneficiamento, que levem em consideração a categoria de peso, destreza do filetador e tempo de filetagem empregado para cada método.

\section{Conclusões}

O método de filetagem no qual se retira a pele do peixe inteiro e depois remove o filé (IPF) proporcionou os melhores resultados de rendimento de filé e músculo hipaxial profundo. O mesmo método proporcionou bom resultado de pele bruta e limpa e menor porcentagem de resíduos de filetagem.

\section{R. Bras. Zootec., v.31, n.3, p.1076-1084, 2002}




\section{Literatura Citada}

BANZATTO, D.A.; KRONKA, S.N. Experimentação agrícola. 3.ed. Jaboticabal: Fundação Universidade Estadual Paulista, 1995. 247p.

CASTILLO CAMPO, L.F. Situación del comercio de tilapia em el año 2000. Panorama Acuícola, v.6, n.3, p.24-27, 2001.

CLEMENT, S.; LOVELL, R.T. Comparison of processing yield and nutrient composition of culture Nile tilapia (Oreochromis niloticus) and channel catfish (ICTALURUS PUNCTATUS). Aquaculture, v.119, n.2-3, p.299-310, 1994.

CONTRERAS-GUZMÁN, E.S. Bioquímica de pescados e derivados. Jaboticabal: Fundação Universidade Estadual Paulista, 1994. 409p.

CONTRERAS-GUZMÁN, E.S. Bioquímica de pescados e invertebrados. Santiago: Centro de Estudios en Ciencia y Tecnologia de Alimentos - Universidad de Santiago de Chile, 2002. 309p.

FREITAS, J.V.F.; GURGEL, J.J.S. Estudos experimentais sobre a conservação da tilápia do Nilo, Oreochromis niloticus (L. 1766) Trewavas, armazenada no gelo. Boletim Técnico DNOCS, v.42, n.2, p.153-178, 1984.

HILSDORF, A.; PEREIRA, J.L. Perfil de consumo de pescado em restaurantes industriais da região do Vale do Paraíba. Panorama da Aqüicultura, v.9, n.53, p.31-35, 1999.

JORY, D.E.; ALCESTE, C.; CABRERA, T.R. Mercado y comercialización de tilapia en los Estados Unidos de Norteamérica. Panorama Acuícola, v.5, n.5, p.50-53, 2000.

KUBITZA, F. Tilápia: tecnologia e planejamento na produção comercial. Jundiaí: Fernando Kubtiza, 2000. 285p.

KUBITZA, F.; KUBITZA, L.M.M. Qualidade da água, sistemas de cultivo, planejamento da produção, manejo nutricional e alimentar e sanidade. Panorama da Aqüicultura, v.10, n.59, p.44-53, 2000.

MACEDO-VIEGAS, E.M.; SOUZA, M.L.R.; KRONKA, S.N. Estudo da carcaça de tilápia do Nilo (Oreochromis niloticus), em quatro categorias de peso. Revista UNIMAR, v.19, n.3, p.863-870, 1997.

MAKRAKIS, S.; BOMBARDELLI, R.A.; MINEMATSU, R.E. et al. Avaliação do rendimento de filé, pele, vísceras, cabeça e resíduos, utilizando-se diferentes dietas balanceadas na engorda de tilápia (Oreochromis niloticus). In: INTERNATIONAL SYMPOSIUM ON TILAPIA AQUACUlTURE, 5., 2000, Rio de Janeiro. Proceedings... Rio de Janeiro: Ministério da Agricultura e do Abastecimento e Departamento de Pesca e Aqüicultura/Ministério de Agricultura, 2000. v.2, p.435-439.

MARCHI, J.F. Desenvolvimento e avaliação de produtos à base de polpa e surimi produzidos a partir de tilápia Nilótica, Oreochromis niloticus $\boldsymbol{L}$. Viçosa, MG: Universidade Federal de Viçosa, 1997. 85p. Dissertação (Mestrado em Ciência e Tecnologia de Alimentos) - Universidade Federal de Viçosa, 1997.

MARENGONI N.G.; SOUZA M.L.R.; CAÇADOR W.C. Rendimento de filetagem de bagre africano Clarias gariepinus e bagre americano Ictalurus punctatus. In: REUNIÃO ESPECIAL DA SOCIEDADE BRASILEIRA PARA O PROGRESSO DA CIÊNCIA, 6., 1998, Maringá. Anais... Maringá: Sociedade Brasileira para o Progresso da Ciência, 1998. p.523-524.
PEREIRA, K.C.; CAMPOS, A.F.M. Estudo do rendimento da carcaça de tilápia (Oreochromis niloticus), após a obtenção do filé e estudo do aproveitamento do espinhaço para a produção de surimi. In: INTERNATIONAL SYMPOSIUM ON TILAPIA AQUACULTURE, 5., 2000, Rio de Janeiro. Proceedings... Rio de Janeiro: MAA e DPA/MA, 2000. v.2. p.440-445.

REDMAYNE, P. Como el camarón y el salmon de cultivo la tilapia se está conviertiendo rápidamente em un proveedor de filetes frescos y congelados de alta calidad. Virtualmente todos los filetes de tilapia vendidos em los Estados Unidos son importados. Panorama Acuícola, v.5, n.3, p.8-9, 2000.

RIBEIRO, L.P.; LIMA, L.C.; TURRA, E.M. Efeito do peso e do operador sobre o rendimento de filé em tilápia vermelha Oreochromis spp. In: AQUICULTURA BRASIL'98, 1998, Recife. Anais/Proceedings... Recife: SIMBRAQ, 1998. v.2, p.773-778.

SOUZA, M.L.R.; MARANHÃO, T.C.F. Rendimento de carcaça, filé e subprodutos da filetagem da tilápia do Nilo, Oreochromis niloticus (L), em função do peso corporal. Acta Scientiarum, v.23, n.4, p.897-901, 2001.

SOUZA, M.L.R.; MARENGONI, N.G. Processing yield of Nile Tilapia (Oreochromis niloticus) and channel catfish (Ictalurus punctatus). In: CONGRESSO PANAMERICANO DE CIÊNCIAS VETERINÁRIAS, 16., 1998, Santa Cruz de la Sierra, Bolivia. Memorias summary... Santa Cruz de la Sierra: 1998. p.166.

SOUZA, M.L.R.; CASTAGNOLLI, N.; KRONKA, S.N. Influência das densidades de estocagem e sistemas de aeração sobre o peso e características de carcaça da tilápia do Nilo (Oreochromis niloticus). Acta Scientiarum, v.20, n.3, p.387-393, 1998.

SOUZA, M.L.R.; MARENGONI N.G.; PINTO A.A. Rendimento do processamento da tilápia-do-nilo (Oreochromis niloticus): tipos de corte da cabeça em duas categorias de peso. Acta Scientiarum, v.22, n.3, p.701-706, 2000.

SOUZA, M.L.R.; MACEDO-VIEGAS, E.M.; KRONKA, S.N Influência do método de filetagem e categorias de peso sobre rendimento de carcaça da tilápia do Nilo (Oreochromis niloticus) Revista Brasileira de Zootecnia, v.28, n.1, p.1-6, 1999.

VANNUCCINI, S. El enfoque del nuevo mercado de tilapia; en el mundo Occidental. Panorama Acuícola, v.4, n.3, p.22-25, 1999 